American Journal of Environmental Sciences 4 (5): 454-461, 2008

ISSN 1553-345X

(C) 2008 Science Publications

\title{
Recent Trends in Ozone and Particle Concentrations in the Sydney (Australia) Airshed
}

\author{
${ }^{1}$ Merched Azzi and ${ }^{2}$ Hiep Duc \\ ${ }^{1}$ CSIRO Energy Technology, Private Mail Bag 7 Bangor NSW 2234 Australia \\ ${ }^{2}$ NSW Department of Environment and Conservation, PO Box 29 Lidcombe, NSW 2141 Australia
}

\begin{abstract}
An overview is presented of changes in ground level ozone and atmospheric fine particles determined using ambient monitoring data collected at selected locations in Sydney, Australia. After removing seasonally, auto-regressive and moving average dependence from the selected time series, the trend of a selected series was modelled using the fractional long-term dependent component. The selected technique was able to detect the trend at a very small resolution. The method results were compared to the trends obtained using the Rao-Zurbenco method. For selected monitoring stations, between 1980 and 1993, there was a decrease in ozone concentrations followed by a slight increase between 1994 and 2002, and thereafter a stable concentration. Fine particle concentrations as measured by nephelometry, showed a significant decrease from 1980 to 1990, which stabilised beyond 1994.
\end{abstract}

Key words: Long-term ozone data, trend analysis, fine particle, long range dependence method

\section{INTRODUCTION}

Associated with the increasing use of fossil fuels has been an increase in ozone precursors in the atmosphere. Ozone is produced photochemically from these precursor compound,s including reactive organic compounds (ROC), carbon monoxide (CO), in the presence of nitrogen oxides $\left(\mathrm{NO}_{\mathrm{x}}\right)$ and sunlight. For over two decades, ozone concentrations have been monitored at different locations in Sydney, Australia. The recorded monitoring data showed that the National Environment Protection Measure (NEPM) goal for $1 \mathrm{hr}$ ozone of $100 \mathrm{pphm}$ has been violated at different monitoring sites in the Sydney region. Different control strategies to mitigate ozone have been implemented and reductions in the ozone severity have been achieved at a number of locations. At some sites, however, there is still breaching of the NEPM goal.

There are a number of published methods for analysing the trend in ozone concentrations by removing the meteorological effects. These methods ${ }^{[1-3]}$ mainly use different models containing various meteorological variables, which are then removed to isolate the long-term trend component from the other components in the models of the time series. Other recently proposed methods that filter out the different frequency scales to determine the long-term trend, such as the Kolmogorov-Zurbenco filtering approach ${ }^{[4]}$ and the wavelet transform ${ }^{[5,6]}$ have been used on air quality, water and climate data with some success.
One particularly useful method that can be applied to many different air pollutant time series is the Long Range Dependence (LRD) method. This method identifies the LRD component in the fractional ARMA model as the anthropogenic trend ${ }^{[7]}$.

The aim of this study was to present and apply a statistical long-range dependence method to study the trend of ozone and fine particles time series in the Sydney region. This analysis may reveal whether the introduced regulatory programs have been effective in controlling the key air contaminants.

Meteorological effects on air quality: It is well known that meteorology plays a key role in ozone formation and transport. Variations of meteorological conditions at different time scales may impact on ozone levels and may mask any long-term trends that could be attributed to the implementation of control options for selected pollutants. Air masses with different histories can have different ozone concentrations, e.g. ozone levels tend to be higher under hot, sunny conditions. At the same time, elevated temperatures, driven by high solar radiation, cause convection to develop, which in turn can enhance vertical ozone transport. Different statistical models have been used to establish the relationship between some meteorological parameters and surface ozone at urban and remote sites. Among the widely used statistical models, regression modelling which is well defined in physical terms, permits impacting processes to be investigated. 


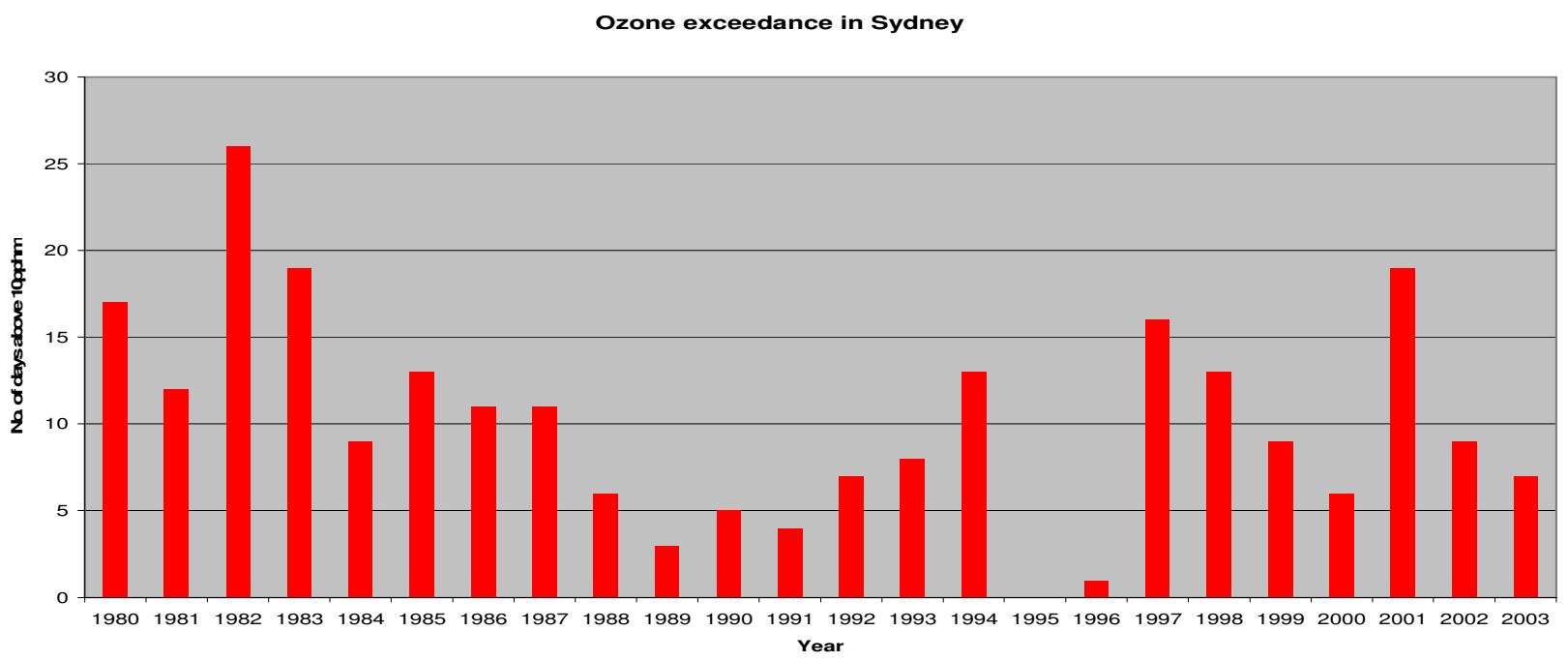

Fig. 1: Number of days above ozone goal (10 pphm)

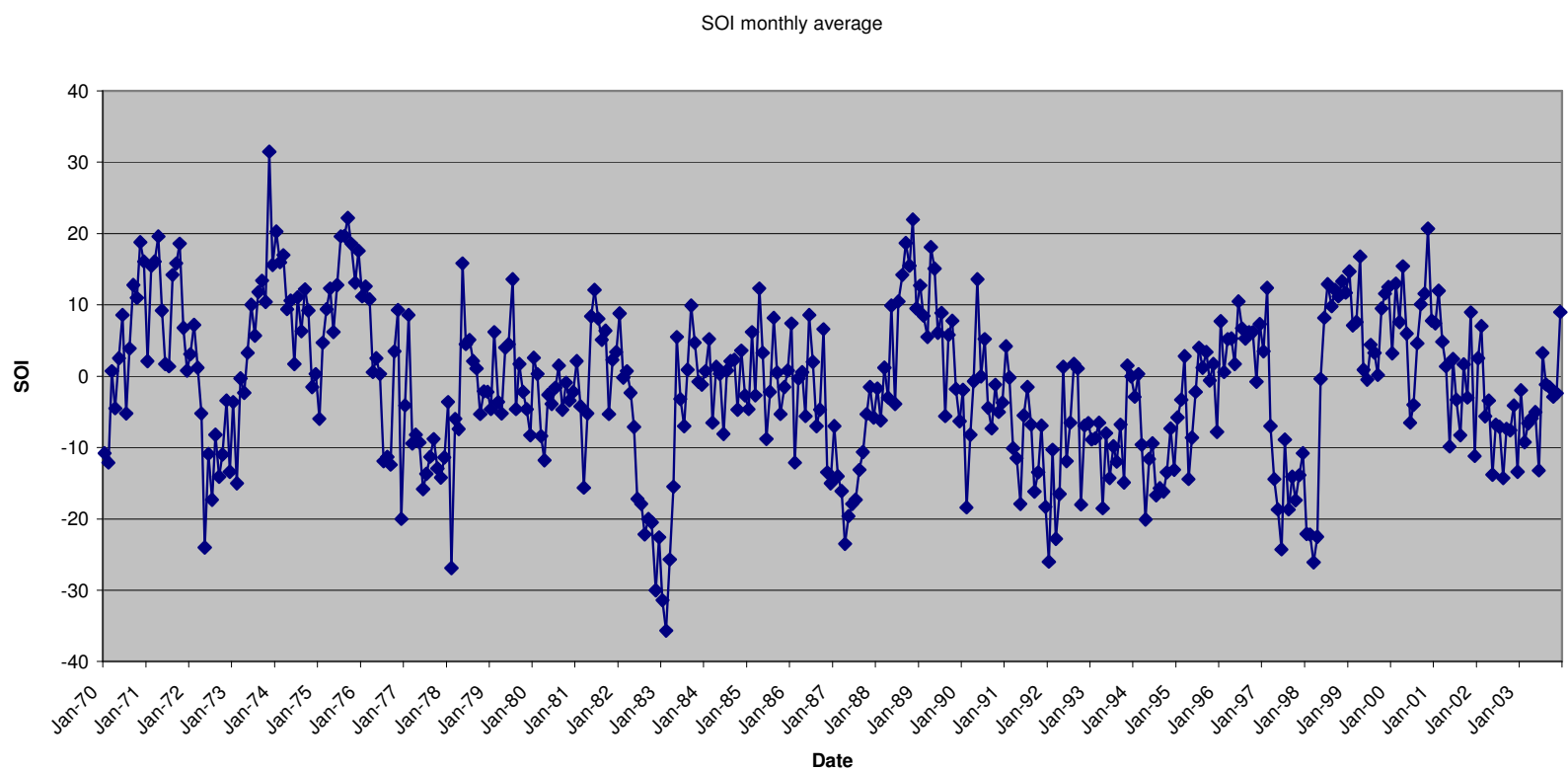

Fig. 2: Monthly average of Southern Oscillation Index (SOI) from 1970 to 2003

These models that incorporate varying degrees of complexity such as temporal cycles, seasonal and meteorological factors and other factors, have been successfully used to detect ozone trends disguised by meteorological conditions ${ }^{[1]}$.

Using ozone concentrations measured between 1980 and 2003, a number of days were selected when ozone concentrations in the Sydney region were above the NEPM goal standard of $10 \mathrm{pphm}$, as illustrated in Fig. 1. The data show that the number of exceedence days in the 1980s is greater than those in the 1990s.
Given the strong effect of temperature on ambient ozone levels, it is informative to look at the various mechanisms that can influence the regional temperature. Besides the diurnal, synoptic and seasonal time scales, there is another longer time global scale driven by the El-Nino Southern Oscillation (ENSO). This phenomenon is strongest in the southern part of the Pacific Ocean. Its effect has caused a drought with high temperatures in Eastern Australia and a high rainfall, cooler climate on the West Coast of North America. 


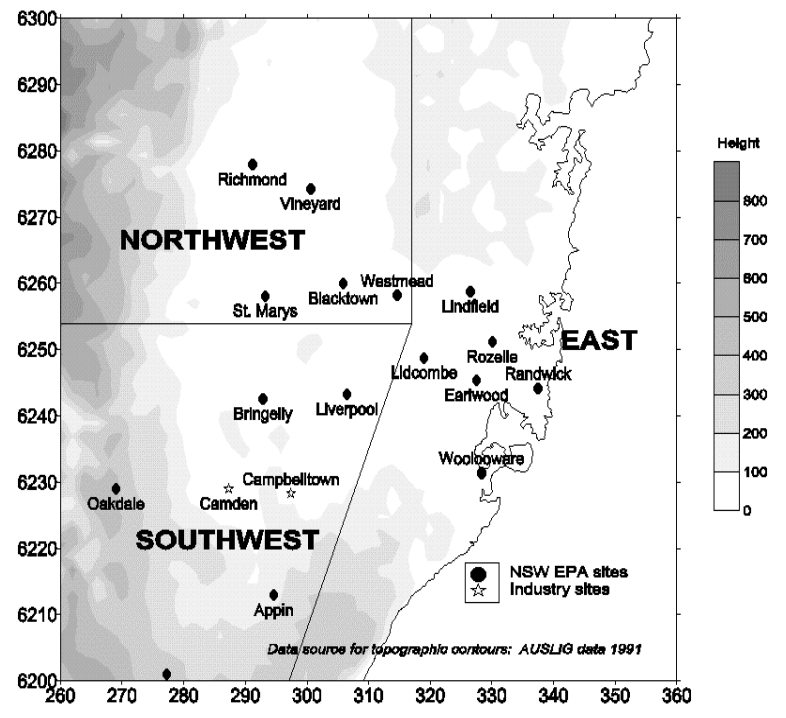

Fig. 3: Sydney air quality monitoring network

Figure 3 shows the monthly average of the Southern Oscillation Index (SOI) from 1970 to 2000. High negative SOI values indicate the El-Nino effect while positive ones, the La-Nina phenomenon. The 3 recent El-Nino phenomena are the 1982-1983, 1987-1988 and 1997-1998 periods. The 1982-1983 period has shown the strongest El-Nino effect. For Sydney, for the periods 1982-1983, 1987-1988 the negative SOI values were associated with high number of days with ozone greater than $10 \mathrm{pphm}$. However, in 2001 and 2002, the SOI values were positive with a high number of days above $10 \mathrm{pphm}$. This may be associated with other phenomena such as bushfires or long-range transport of pollutants from other sources.

It is evident that removing the temperature effect from the ozone concentration time series will significantly reduce the meteorological influence due to natural processes. The remaining effect should be mainly due to anthropogenic sources.

Modelling anthropogenic trends: Two approaches were used to investigate long- term changes in to gain insight into the effectiveness of emissions control strategies implemented in the Sydney airshed.

Rao-Zurbenco method. Rao and Zurbenco ${ }^{[1]}$ used the ozone and temperature time series to find the meteorologically adjusted ozone trends by using filtering and regression techniques. A time series $X(t)$ is assumed to be represented as:

$$
X(t)=e(t)+S(t)+W(t)
$$

Where $e(t)$ is the trend component, $S(t)$ the seasonal component and $W(t)$ white noise. The random variations $W(t)$ can be removed from the series by a simple iterative application of a moving average filter:

$$
Y(i)=\frac{1}{m} \sum_{j=-k}^{k} X(i+j), m=2 k+1,
$$

Where $Y(i)$, the output of the first iteration, then becomes the input for the next iteration of (2). The number of iterations $(p)$ and the filter width value $m$ are to be determined from the data to achieve noise-free series. This p-iterative application of a moving average filter of width $\mathrm{m}$, is called the Kolmogorov-Zurbenko filter, $\mathrm{KZ}(\mathrm{m}, \mathrm{p})$.

The effect of meteorological variability on the air pollutant time series has to be removed prior to any trend analysis. In the Rao-Zurbenko method for a daily ozone series, the meteorological effects are represented by the maximum daily temperature. Both the ozone and the temperature time series are first filtered to remove the noise using the Kolmogorov-Zurbenco filter, $\mathrm{KZ}(\mathrm{m}, \mathrm{p})$. The meteorological effects can then be removed by using the regression technique.

To be specific, denote the filtered log of ozone concentrations by $O_{k-z}(t)$ and the filtered temperature by $T_{k z}(t)$, then the meteorological effects, represented by the seasonal component, are removed from the filtered $\log$ of ozone by the linear regression:

$O_{k z}(t)=a+b T_{k z}(t)+\varepsilon(t)$

The noise term $\varepsilon(t)$ then represents changes in ozone attributable to changes in emissions.

The Rao-Zurbenco method can be applied to any pollutant that exhibits a temperature or seasonal dependence. However, another method,the Long Range Dependence (LRD) model, can be applied to any longterm time series.

Modelling the Long-range Dependence (LRD) component: The recent literature on air pollution modelling has paid attention to the long-range dependence in air quality data. It has now been established that the LRD phenomenon is present in air quality, meteorological, hydrological and geophysical data $^{[8-10]}$.

A stochastic process $X(t)$ is said to exhibit LRD if its spectral density has the form

$f(\omega)=f_{*}(\omega) \omega^{-2 \beta}, \quad \beta>0, \omega \in \Re$,

where $f_{*}(\omega)$ is slowly varying as $\omega \rightarrow 0$. The spectral density has an integrable singularity at the 
origin if $0<\beta<1 / 2$ with the characteristic effect that the autocovariance function of $X(t)$ decays to zero at a very slow rate so that the autocorrelation function is not absolutely summable.

The significant component at a very low frequency shows that the time series contains a slow- varying trend, which is not easily detected and removed using standard time series analysis such as autoregressive and moving average (ARMA) or autoregressive inegrated moving average (ARIMA). In fact, the presence of LRD invalidates many of the traditional methods of data description using autoregressive and moving ARMA models $^{[8]}$.

A discrete stationary approximation of the LRD factor $\omega^{-2 \beta}$ of (4) is

$f(\omega)=\frac{\sigma^{2}}{2 \pi} \frac{1}{\left|1-e^{i \omega}\right|^{2 d}}, \sigma^{2}>0,0<d<1 / 2, \omega \in(-\pi, \pi]^{[7]}$

The LRD and short-memory components of a discrete time series $X(t)$ can therefore be modelled by a fractional ARMA $(p, d, q)$ :

$$
\begin{aligned}
& (1-B)^{d}\left(1-\theta_{1} B-\ldots-\theta_{p} B^{p}\right) X(t)= \\
& \left(1+\phi_{1} B+\ldots+\phi_{q} B^{q}\right) \mathcal{E}(t)
\end{aligned}
$$

where $B$ is the backshift operator $B X(t)=X(t-1), d$ is the LRD parameter, $\mathcal{E}(t)$ is white noise with variance $\sigma^{2}$.

The spectral density of the time series generated by model (5) is

$f(\omega)=\frac{\sigma^{2}}{2 \pi} \frac{1}{\left|1-e^{i \omega}\right|^{2 d}} \frac{\left|1-\phi_{1} e^{i \omega}-\ldots-\phi_{q} e^{i q \omega}\right|^{2}}{\left|1-\theta_{1} e^{i \omega}-\ldots-\theta_{p} e^{i p \omega}\right|^{2}}, \omega \in(-\pi, \pi]$

The Sydney pollutant series appear to be additive seasonally, suggesting a model of the form

$$
X(t)=S(t)+R(t)
$$

where $S(t)$ is the seasonal component and $R(t)$ is the random component. Also, due to large variations in the seasonal component, particularly in the summer period, it is necessary to use a Box-Cox transform

$$
Y(t)=\frac{X^{\alpha}(t)-1}{\alpha}, \alpha>0
$$

to stabilise the variance. A special form of the Box-Cox transform is the logarithmic transform as $\alpha \rightarrow 0$.

The average of the Box-Cox transform of the daily maxima over all years for each day of the year is then regressed on a set of annual harmonics. Substraction of the estimated seasonal effect from the Box-Cox transform of the daily maxima then yields the seasonally adjusted series ready for trend analysis. The series is seasonally adjusted using the yearly profile of the transformed series. For the ozone and nitrogen oxides data series in Sydney, the choice of $\alpha=0.2$ based on (6) has been proved as appropriate ${ }^{[7]}$.

The Haslett-Raftery algorithm ${ }^{[10]}$ can be invoked to estimate $d$ and the ARMA coefficients of (5) simultaneously on the seasonally adjusted series. Removing the short-memory ARMA component from the estimated model (5) will then give the LRD component for trend analysis.

Application of LRD modelling to ozone and particles time series: Time series of monitoring data for ozone and particles (Nephelometer, PM10 and total suspended particulates (TSP)) collected at a selected monitoring stations, were used to study their trends using the LRD method. Prior to 1993, monitoring stations were mostly located to the east of Sydney, with very in the northwest and south-west regions. In 1998, the Sydney monitoring station network was extended to accommodate 19 monitoring stations located throughout the Sydney region as illustrated in Fig. 4. Air pollutants and meteorological parameters are measured continuously and then consolidated and stored as hourly values.

The daily maximum values for ozone and particles are used in the trend analysis. Monitoring data has been assessed for missing data and quality assured for accuracy and calibration. Missing data are either interpolated (less than 3 missing points) or replaced with average seasonally values in the series. The ozone and nephelometer data are measured each hour across many sites in the Sydney basin while the $\mathrm{PM}_{10}$ and TSP data are collected once every six-day at only some sites in the east of Sydney near the centre of the city.

Time series data were transformed using Box-Cox Transform to stabilise the variance before being analysed to find the trends. The modelling of the LRD component on the ozone and nephelometer data, after removing the seasonally variation, indicates that each of the series can be represented by an autoregressive (AR) model of order 3, a moving average (MA) of order 1 and a long range dependence (as represented by a fractional coefficient) component.

The $\mathrm{PM}_{10}$ and TSP data, recorded once every six days, did not exhibit high variation. There is no need to transform the data and the seasonality component was not modelled. For 6-day cycled $\mathrm{PM}_{10}$ and TSP data sets, 

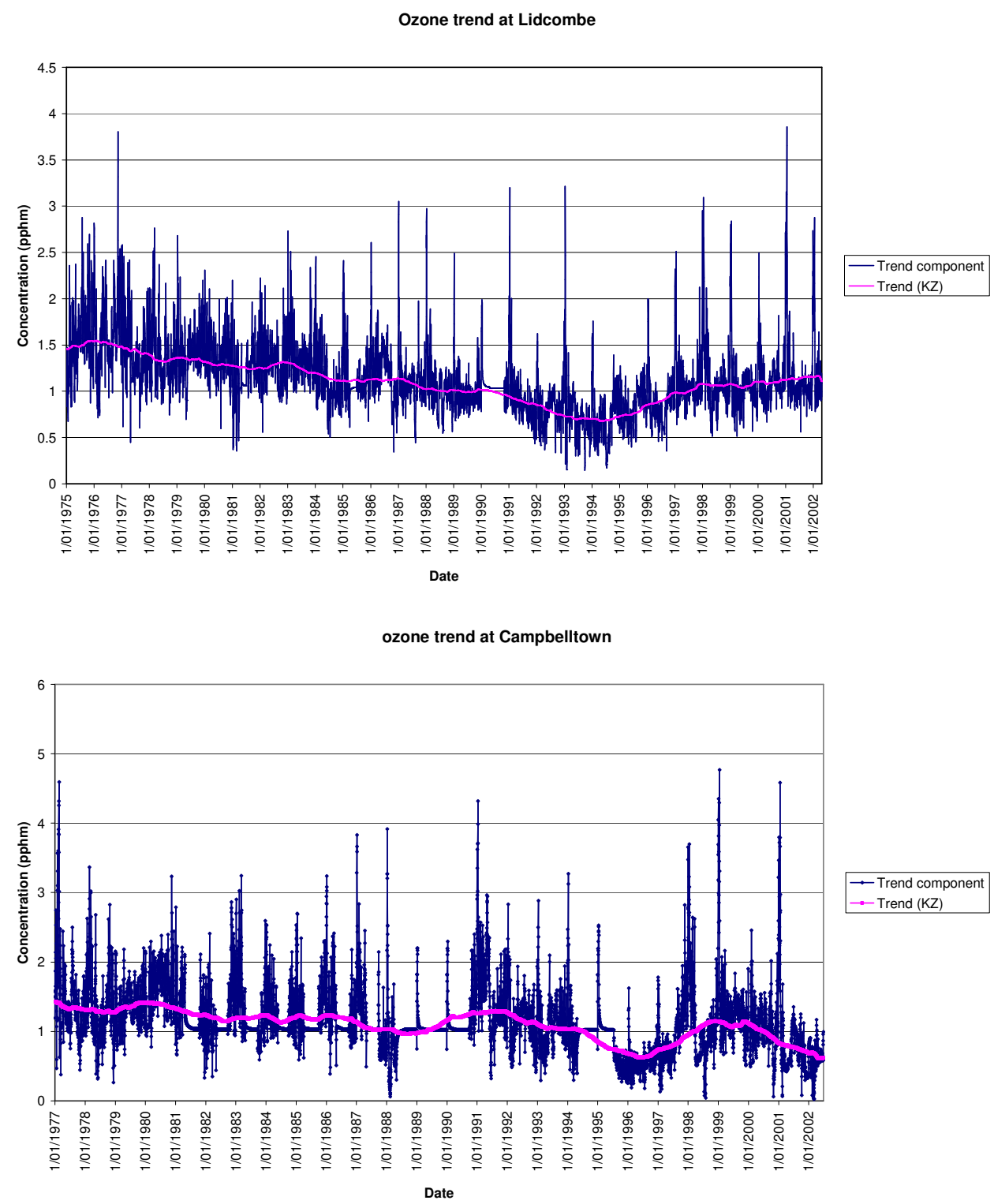

Fig. 4: Ozone trends at Lidcombe and Campbelltown sites

the series exhibit an autoregressive component of order 1 and a MA component of the same order.

The trend part of the time series is considered to be the LRD component. To see the best trend pattern, a smoothing process using Kolmogorov-Zurbenco (KZ) filter Rao, Zurbenco ${ }^{[1]}$ and Rao, Zurbenco et al. ${ }^{[4]}$ with 450 data points, $\operatorname{KZ}(450,1)$, were applied to ozone trend components and $\mathrm{KZ}$ filter, $\mathrm{KZ}(50,1)$, with 50 data points applied to $\mathrm{PM}_{10}$ and TSP trend components.
The smoothed trends of ozone, $\mathrm{PM}_{10}$ and TSP for selected sites are shown in Fig. 4. For all trend graphs except those of $\mathrm{PM}_{10}$ and TSP, the trend values were obtained after an inverse transform of the transformed series. The trend values are relative to the long-term average value indicated by the unit value of 1 .

As noted by Thompson et al. ${ }^{[1]}$, summarising nonlinear trends beyond a graphical display is difficult and complicated, as it cannot be captured by measures such as percent change per year or per decade. 


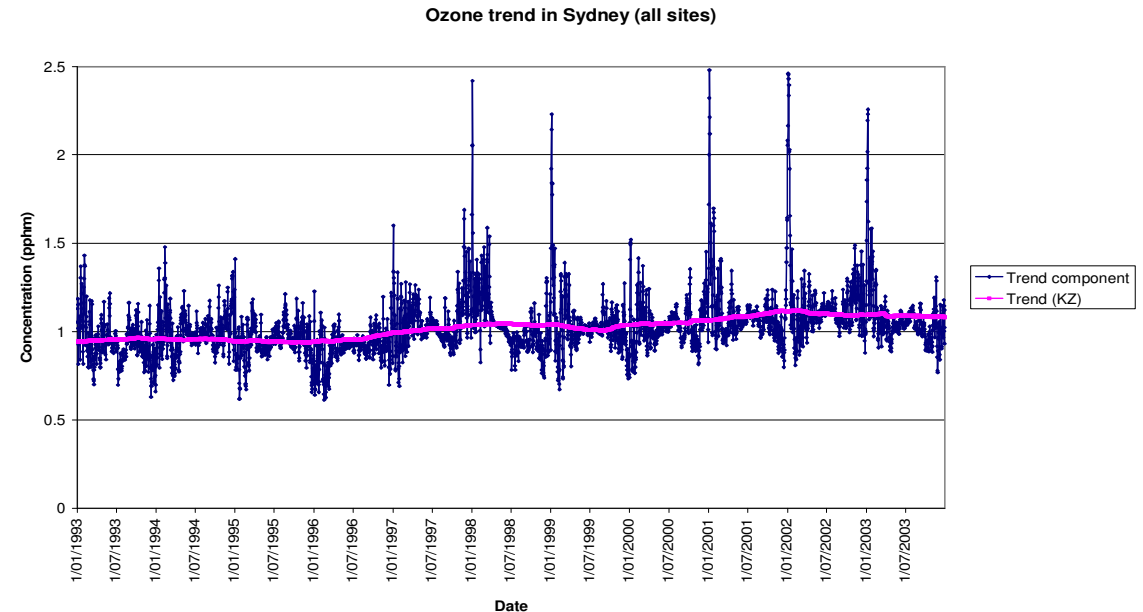

Fig. 5: Ozone trend in Sydney
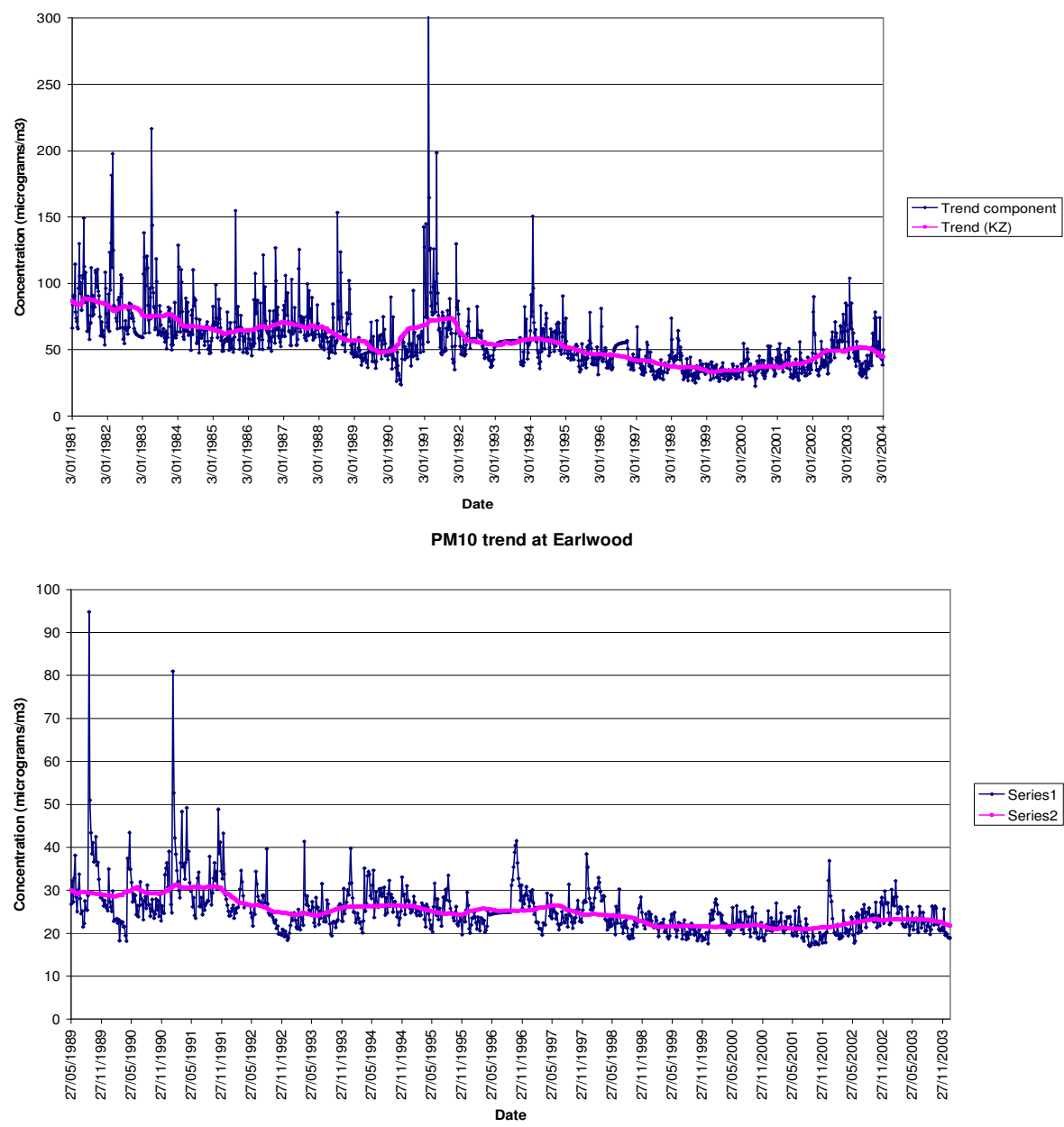

Fig. 6: TSP and PM10 trends at Earlwood. Series 1 refers to the trend component and series 2 refers to trend (KZ) 


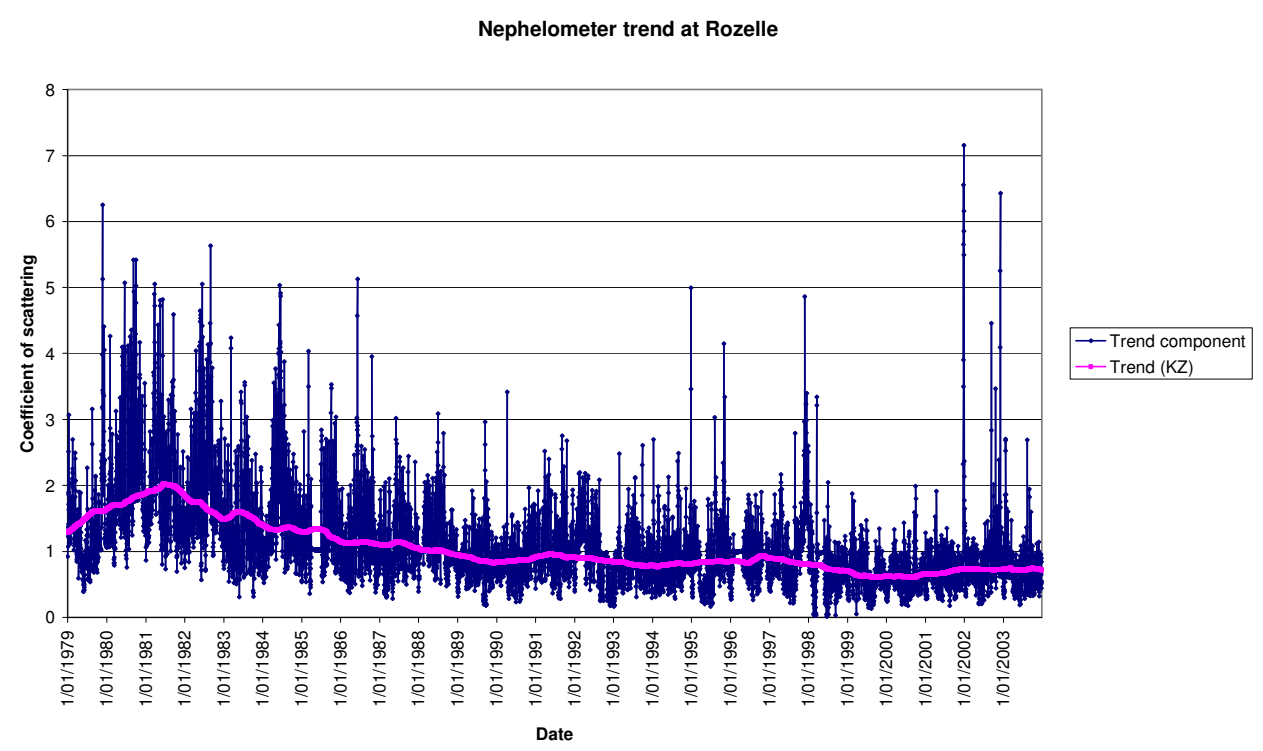

Fig. 7: Fine particles (as measured by Nephelometer) trend at Rozelle

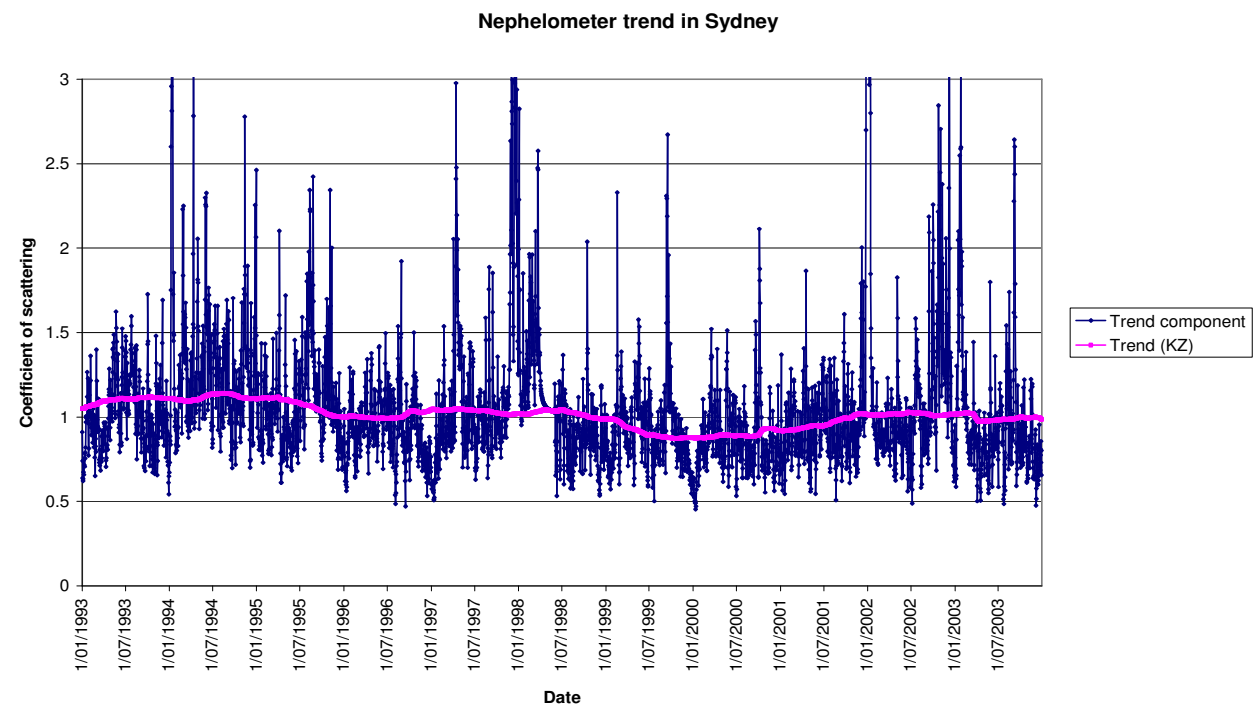

Fig. 8: Fine particles (as measured by nephelometer) trend in Sydney

Yet, some form of summary measure is necessary to better describe the trend. Qualitative summary description is described below over some periods in which nonlinear trends are monotone.

Between 1975 and 1994, the calculated trend of the ozone time series at the Lidcombe site Fig. 3 shows a general decrease in the ozone levels at this location. However, beyond 1994, an increase in the ozone trend was exhibited at the selected site. Using data from the Campbelltown monitoring site Fig. 3, the trend is similar to that at Lidcombe, except that after 2000, a decrease of ozone is observed.
For the Sydney region, a combined daily maximum series of ozone and nephelometer data representing the whole region was obtained by using the maximum daily value from daily maximum measurements from all the available sites. The trend from this pollutant series can be considered as the overall trend for the region. However, as the spatial scale variability has been implicitly filtered or averaged out in the combined process, the change in trend curve will be much smaller than at individual sites. The overall trend from the ozone data representing all the sites in the Sydney region (1993-2003) is illustrated in Fig. 5. A relatively 
stable pattern of the ozone trend was calculated for the Sydney region. Beyond 1997, the calculated ozone trend exhibited a slight increase in the ozone levels over the Sydney region.

The analysis of fine particle data to determine the trend over the selected period was carried out using monitoring data from available sites in the Sydney region. Between 1981 and 2004, the analysis of the Earlwood site TSP data showed a general downward trend. However, between 1990 and 1992, an increase in the TSP trend was detected. Between 2001 and 2004, the detected TSP trend was shifting towards the increase. At Earlwood, the detected PM10 trend exhibited a reduction pattern for the PM10 levels. However, between 2001 and 2003 a slight increase in the PM10 trend levels was detected Fig. 6.

At Rozelle, fine particles as measured by nephelometry, showed a significant decrease from mid1980 to mid-1990 as illustrated in Fig. 7. Beyond 1999, the data analysis showed a stabilised trend.

The calculated trend of fine particles measured by nephelometry for the Sydney region is illustrated in Fig. 8. Between 1993 and 2001, a general downward trend was detected for the Sydney region. After 2001, the data exhibited a slight increase in the calculated trend for the Sydney region.

\section{DISCUSSION AND CONCLUSION}

The LRD model for air pollution time series has been applied to detect the trend of ozone and fine particles time series at selected monitoring sites in the Sydney basin. For fine particles (under $2.5 \mu \mathrm{m}$ diameter), the calculated trend showed a clear decrease in concentration that reached a minimum in 2000. Beyond 2000, the calculated trend have shown a slight increase in the fine particle concentrations.

The ozone time series analysis showed that between 1993 and 2002, the calculated ozone trend exhibited a general increase in ozone, and beyond 2002 a general stable trend. Any attempt to assess the changing behaviour of ozone requires further knowledge about the type and timing of implemented control strategies for ozone mitigation. From a photochemical point of view, this could be explained by an increasing emission of volatile organic compounds (VOC) across the Sydney basin or a decreasing level of nitrogen oxides where the extent of reaction is less than an optimum value.

There is a limitation with the ability of the LRD method to find an air quality trend due to anthropogenic sources free from meteorological effects. In most situations where the data period for analysis is usually about 10 (or $>10$ ) years, it is effective in isolating and removing short-term climate variability on the seasonal and inter-annual scales. But for long-term climate changes (such as the global warming on inter-decade scale), it may not be possible to separate these sources of climatic variability as they are at about or below the lowest frequency range that can be resolved with the time window of the available data. In other words, they are at about the same lowest frequency that can be attributed to the anthropogenic sources. However, some of the anthropogenic activities can affect and change the local climate on the shorter long-term scale (such as the urban-heat island effects in large cities). In this case, this anthropogenic source is then accounted for and is included in the LRD component of the time series.

\section{REFERENCES}

1. Rao, S.T. and I.G. Zurbenko, 1994. Detecting and tracking changes in ozone air quality. J. Air and Waste Manag. Assoc., 44: 1089-1092.

2. Flaum J., S. Rao and I. Zurbenco, 1996. Moderating the influence of meteorological conditions on ambient ozone concentrations. J. Air and Waste Manag. Assoc., 46: 35-46.

3. Xu, D., D. Yap and P. Taylor, 1996. Meteorologically adjusted ground level ozone trends in Ontario. Atmosph. Environ., 30: 1117-1124.

4. Rao, S. T., I.G. Zurbenco, R. Neagu, P.S. Porter, J.Y. Yu and R.F. Henry 1997. Space and time scales in ambient ozone data. Bull. Am. Meteorolog. Soc., 78: 2153-2166.

5. Whitcher, B., 2000. Wavelet analysis of seasonal long memory. In V. Núñoz-Antón and E. Ferreira (Eds.) Statistical Modelling, Proc. 15th Int. Workshop Stat. Modelling, pp: 276-281, Bilbao, Spain: Servicio Editorial de la Universidad del País Vasco.

6. Lau, K.M. and H. Weng, 1995. Climate signal detection using wavelet transform: how to make a time series sing. Bull. Am. Meteorolog. Soc., 76: 2391-2402.

7. Anh, V., H. Duc and M. Azzi, 1997. Modelling anthropogenic trends in air quality data. J. Air and Waste Manag. Assoc., 47: 66-71.

8. Beran, J. 1992. Statistical models for data with longrange dependence., Stat. Sci., 4: 404-427.

9. Ooms, M. and P. Franses, 2001. A seasonal periodic long memory model for monthly river flows. Environ. Model. Software, 16: 559-569.

10. Haslett, J. and A. Raftery, 1989. Space-time modelling with long-memory dependence: Assessing Ireland's wind power resource. Appl. Stat., 38: 1-50.

11. Thompson, M., J. Reynolds, L. Cox, P. Guttorp and P. Sampson, 2001. A review of statistical methods for the meteorological adjustment of tropospheric ozone. Atmosph. Environ., 35: 617-630. 